Article

\title{
Picture Fuzzy Petri Nets for Knowledge Representation and Acquisition in Considering Conflicting Opinions
}

\author{
Xue-Guo Xu ${ }^{1}$, Hua Shi ${ }^{1}$, Dong-Hui Xu ${ }^{1, *}$ and Hu-Chen Liu ${ }^{2,3}$ \\ 1 School of Management, Shanghai University, Shanghai 200444, China; xuxueguo@shu.edu.cn (X.-G.X.); \\ shihua1980@shu.edu.cn (H.S.) \\ 2 College of Economics and Management, China Jiliang University, Hangzhou 310018, China; \\ huchenliu@shu.edu.cn \\ 3 School of Economics \& Management, Tongji University, Shanghai 200444, China \\ * Correspondence: xudonghui@shu.edu.cn
}

Received: 29 January 2019; Accepted: 1 March 2019; Published: 8 March 2019

check for updates

\begin{abstract}
Fuzzy Petri nets (FPNs) have been applied in many fields as a potential modeling tool for knowledge representation and reasoning. However, there exist many deficiencies in the conventional FPNs when applied in the real world. In this paper, we present a new type of FPN, called picture fuzzy Petri nets (PFPNs), to overcome the shortcomings and improve the effectiveness of the traditional FPNs. First, the proposed PFPN model adopts the picture fuzzy sets (PFSs), characterized by degrees of positive membership, neutral membership, and negative membership, to depict human expert knowledge. As a result, the uncertainty, due to vagueness, imprecision, partial information, etc., can be well-handled in knowledge representation. Second, a similarity degree-based expert weighting method is offered for consensus reaching processes in knowledge acquisition. The proposed PFPN model can manage the conflicts and inconsistencies among expert evaluations in knowledge parameters, thus, making the obtained knowledge rules more accurate. Finally, a realistic example of a gene regulatory network is provided to illustrate the feasibility and practicality of the proposed PFPN model.
\end{abstract}

Keywords: fuzzy Petri net (FPN); picture fuzzy set (PFS); knowledge representation; conflict opinion; expert system

\section{Introduction}

In expert systems, fuzzy Petri nets (FPNs) are often utilized as a potential modeling tool for knowledge representation and reasoning [1,2]. The combination of the graphical power of Petri nets and ability of fuzzy sets to express vague information makes FPNs suitable for modeling uncertain rule-based expert systems [3,4]. An FPN is a marked graphical system containing places and transitions [5]. Due to the capacity to depict imprecise knowledge and support inference processes, FPNs have garnered an increasing interest in both academics and practitioners and have been used in a lot of fields, such as fault diagnosis [6,7], adaptive software systems modeling [8], reliability optimization design [9], genetic regulatory network design [10], and DNA sequencing prediction [11,12].

However, the conventional FPNs have many deficiencies, as pointed out in previous studies $[13,14]$. Some scholars criticized the limitation of FPNs in knowledge representation when the knowledge parameter values are restricted to be crisp values between 0 and 1 . To address this issue, some uncertainty methods have been incorporated into FPNs. For example, Chen [1] represented the truth values of propositions, the weights of propositions, and the certainty factors of rules by 
fuzzy numbers in a weighted FPN model. Linguistic interval 2-tuples were adopted by Li et al. [15] to acquire and represent tacit knowledge in the interval-valued intuitionistic FPNs. Liu et al. [16] proposed a new type of FPN for knowledge representation based on intuitionistic fuzzy set (IFS) theory. A generalized weighted FPN model, where the truth values and the weights of places were represented by intuitionistic fuzzy numbers, was proposed by Suraj [17]. However, due to the complexity and uncertainty involved, there exist many circumstances in which it is improper to use fuzzy sets or IFSs to accurately express experts' knowledge. Recently, the concept of picture fuzzy sets (PFSs) was proposed by Cuong [18] to describe uncertain phenomena and information. The PFSs, characterized by three membership degrees: positive, neutral, and negative, are more appropriate to deal with human expert evaluation information. Since its introduction, the PFS has received widespread attention in academia and has been applied in many practical fields [19-22].

Knowledge acquisition plays a significant role in the development of a rule-based expert system. But there are few studies considering the acquisition of knowledge parameters of FPNs in the literature. With the increasing complexity of expert systems, difficulties arise during development, particularly when acquiring knowledge from human experts. It is an even much more difficult problem to fine-tune the knowledge parameters of fuzzy production rules (FPRs) during the knowledge base update stage [23,24]. Consequently, the traditional process to extract FPRs from experts can be a bottleneck, causing delay in establishing an expert system [25,26]. In this respect, Liu et al. [24] suggested a knowledge acquisition approach using a fuzzy evidential reasoning approach. Liu et al. [27] proposed a group decision-making approach using hesitant 2-tuple linguistic term sets to derive the initial truth values of FPNs. Li et al. [15] developed a theoretical model based on linguistic interval 2-tuples and interval-valued intuitionistic FPNs (IVIFPNs) for acquiring and representing tacit knowledge in a healthcare organization. On the other hand, domain experts can have many differences in terms of their backgrounds, attitudes, and organizations, and conflicts are inevitable. Consequently, it is important to develop methods to effectively handle conflicts, which will lead to efficiency improvements in knowledge acquisition processes.

Based on the above analyses, the objective of this paper is to propose a new type of FPN, called picture fuzzy Petri nets (PFPNs), to represent and acquire imprecise and uncertain expert knowledge. First, we apply PFSs to quantitatively represent the complex and uncertain knowledge in constructing an expert system. This application will provide a practical, effective tool to describe uncertain information gathered in knowledge representation. Second, we use a similarity degree-based method to determine the importance weights of experts in knowledge acquisition. This adaption can take full consideration of conflicting and inconsistent opinions among experts, and it can facilitate consensus-reaching processes. In addition, a case study about the gene regulatory network is given to demonstrate the application, practicality, and effectiveness of the proposed PFPN model. The remainder of this article is organized as follows: Section 2 introduces the basic concepts of PFSs briefly. The definitions of FPNs and FPRs are given in Section 3. Section 4 develops the PFPN model, and Section 5 presents a realistic example. Finally, Section 6 draws conclusions of this article.

\section{Preliminaries}

\subsection{Picture Fuzzy Sets}

Cuong [18] pioneered the concept of PFSs, which are a direct extension of fuzzy sets [28] and IFSs [29]. Its definition is given as follows:

Definition 1 [18]. The form of a PFS $\widetilde{A}$ on a universe $X$ is:

$$
\widetilde{A}=\left\{\left(x, \mu_{\widetilde{A}}(x), \eta_{\widetilde{A}}(x), v_{\widetilde{A}}(x)\right) \mid x \in X\right\},
$$

where $\mu_{\widetilde{A}}(x) \in[0,1]$ means the degree of positive membership of $x$ in $\widetilde{A}, \eta_{\widetilde{A}}(x) \in[0,1]$ means the degree of neutral membership of $x$ in $\widetilde{A}, v_{\widetilde{A}}(x) \in[0,1]$ means the degree of negative membership of $x$ 
in $\widetilde{A}$, and $\mu_{\widetilde{A}}, \eta_{\widetilde{A}}$, and $v_{\widetilde{A}}$ satisfy the following conditions: $0 \leq \mu_{\widetilde{A}}(x)+\eta_{\widetilde{A}}(x)+v_{\widetilde{A}}(x) \leq 1(\forall x \in X)$. Then, $\pi_{\widetilde{A}}(x)=1-\left(\mu_{\widetilde{A}}(x)+\eta_{\widetilde{A}}(x)+v_{\widetilde{A}}(x)\right)$ could be called the degree of refusal membership of $x$ in $\widetilde{A}$.

For convenience, $\widetilde{a}=(\mu, \eta, v)$ is called a picture fuzzy number (PFN), where $\mu(x), \eta(x)$ and $v(x)$ satisfy the following conditions: $\mu(x) \in[0,1], \eta(x) \in[0,1], v(x) \in[0,1]$ and $\mu(x)+\eta(x)+v(x) \leq 1$.

Definition $2[30,31]$. Let $\widetilde{a}=(\mu, \eta, v)$ be a PFN, then a score function $S$ of $\widetilde{a}$ can be represented as follows:

$$
S(\widetilde{a})=\mu_{1}-v_{1}, S(\widetilde{a}) \in[-1,1] .
$$

Definition $3[30,31]$. Let $\widetilde{a}=(\mu, \eta, v)$ be a PFN, then an accuracy function $H$ of $\widetilde{a}$ can be represented as follows:

$$
H(\widetilde{a})=\mu_{1}+\eta_{1}+v_{1}, H(\widetilde{a}) \in[0,1],
$$

where $H(\widetilde{a})$ is associated with the degree of accuracy of the PFN, which means that the larger the value of $H(\widetilde{a})$, the more the accuracy of $\widetilde{a}$.

Definition 4 [31]. Given two PFNs $\widetilde{a}_{1}=\left(\mu_{1}, \eta_{1}, v_{1}\right)$ and $\widetilde{a}_{2}=\left(\mu_{2}, \eta_{2}, v_{2}\right)$, some operational laws of PFNs can be defined as follows:

(1) $\widetilde{a}_{1} \oplus \widetilde{a}_{2}=\left(1-\left(1-\mu_{1}\right)\left(1-\mu_{2}\right), \eta_{1} \eta_{2},\left(v_{1}+\eta_{1}\right)\left(v_{2}+\eta_{2}\right)-\eta_{1} \eta_{2}\right) ;$

(2) $\widetilde{a}_{1} \otimes \widetilde{a}_{2}=\left(\left(\mu_{1}+\eta_{1}\right)\left(\mu_{2}+\eta_{2}\right)-\eta_{1} \eta_{2}, \eta_{1} \eta_{2}, 1-\left(1-v_{1}\right)\left(1-v_{2}\right)\right)$;

(3) $\lambda \widetilde{a}_{1}=\left(1-\left(1-\mu_{1}\right)^{\lambda}, \eta_{1}^{\lambda},\left(v_{1}+\eta_{1}\right)^{\lambda}-\eta_{1}^{\lambda}\right), \lambda>0$;

(4) $\tilde{a}_{1}^{\lambda}=\left(\left(\mu_{1}+\eta_{1}\right)^{\lambda}-\eta_{1}{ }^{\lambda}, \eta_{1}{ }^{\lambda}, 1-\left(1-v_{1}\right)^{\lambda}\right), \lambda>0$.

Definition 5 [19]. Given two PFNs $\widetilde{a}_{1}=\left(\mu_{1}, \eta_{1}, v_{1}\right)$ and $\widetilde{a}_{2}=\left(\mu_{2}, \eta_{2}, v_{2}\right)$, and based on the score function $S(\widetilde{a})$ and the accuracy function $H(\widetilde{a})$, the order relation of $a_{1}$ and $a_{2}$ is defined as follows:

(1) If $S\left(\widetilde{a}_{1}\right) \leq S\left(\widetilde{a}_{2}\right)$, the $\widetilde{a}_{1}$ is smaller than $\widetilde{a}_{2}$, defined as $\widetilde{a}_{1}<\widetilde{a}_{2}$;

(2) If $S\left(\widetilde{a}_{1}\right)=S\left(\widetilde{a}_{2}\right)$, then,

(1) If $H\left(\widetilde{a}_{1}\right)=H\left(\widetilde{a}_{2}\right), \widetilde{a}_{1}$ and $\widetilde{a}_{2}$ indicate the same information, defined as $\widetilde{a}_{1}=\widetilde{a}_{2}$;

(2) If $H\left(\widetilde{a}_{1}\right)<H\left(\widetilde{a}_{2}\right), \widetilde{a}_{1}$ is smaller than $\widetilde{a}_{2}$, defined as $\widetilde{a}_{1}<\widetilde{a}_{2}$.

Definition 6 [31]. Let $\widetilde{a}_{j}=\left(\mu_{j}, \eta_{j}, v_{j}\right)(j=1,2, \ldots, n)$ be a collection of PFNs, then the picture fuzzy weighted geometric (PFWG) operator is defined as:

$$
\operatorname{PFWG}\left(\widetilde{a}_{1}, \widetilde{a}_{2}, \ldots, \widetilde{a}_{n}\right)=\prod_{j=1}^{n} \widetilde{a}_{j}^{w_{j}},
$$

where $w=\left(w_{1}, w_{2}, \ldots, w_{n}\right)^{T}$ is the weight vector of $\left\{\widetilde{a}_{1}, \widetilde{a}_{2}, \ldots, \widetilde{a}_{n}\right\}$, with $w_{j} \in[0,1]$ and $\sum_{j=1}^{n} w_{j}=1$. The aggregated value calculated by the PFWG operator is also a PFN, expressed as:

$$
\operatorname{PFWG}\left(\widetilde{a}_{1}, \widetilde{a}_{2}, \ldots, \widetilde{a}_{n}\right)=\left\langle\prod_{j=1}^{n}\left(\mu_{j}+\eta_{j}\right)^{w_{j}}-\prod_{j=1}^{n} \eta_{j}^{w_{j}}, \prod_{j=1}^{n} \eta_{j}^{w_{j}}, 1-\prod_{j=1}^{n}\left(1-v_{j}\right)^{w_{j}}\right\rangle .
$$


Definition 7 [20]. Given two PFNs $\widetilde{a}_{1}=\left(\mu_{1}, \eta_{1}, v_{1}\right)$ and $\widetilde{a}_{2}=\left(\mu_{2}, \eta_{2}, v_{2}\right)$, the relative projection of $\widetilde{a}_{1}$ on $\widetilde{a}_{2}$, which describes the closeness of $\widetilde{a}_{1}$ on $\widetilde{a}_{2}$, is defined as:

$$
R P_{\widetilde{a}_{2}}\left(\widetilde{a}_{1}\right)=\frac{\mu_{1} \mu_{2}+\eta_{1} \eta_{2}+\left(1-v_{1}\right)\left(1-v_{2}\right)}{\mu_{2}^{2}+\eta_{2}^{2}+\left(1-v_{2}^{2}\right)} .
$$

Definition 8 [20]. Let us suppose that $\widetilde{R}^{1}=\left(\widetilde{r}_{i j}^{1}\right)_{m \times n}=\left(\mu_{i j}^{1}, \eta_{i j}^{1}, v_{i j}^{1}\right)_{m \times n}$ and $\widetilde{R}^{2}=\left(\widetilde{r}_{i j}^{2}\right)_{m \times n}=$ $\left(\mu_{i j}^{2}, \eta_{i j}^{2}, v_{i j}^{2}\right)_{m \times n}$ are two picture fuzzy evaluation matrices, then the relative projection of $\widetilde{R}^{1}$ on $\widetilde{R}^{2}$, which describes the closeness of $\widetilde{R}^{1}$ on $\widetilde{R}^{2}$, is defined as:

$$
R P_{\widetilde{R}^{2}}\left(\widetilde{R}^{1}\right)=\frac{1}{m n} \sum_{i=1}^{m} \sum_{j=1}^{n} \frac{\left|R P_{\widetilde{r}_{i j}^{2}}\left(\widetilde{r}_{i j}^{1}\right)-1\right|-\min _{1 \leq i \leq m, 1 \leq j \leq n}\left\{\left|R P_{\widetilde{r}_{i j}^{2}}\left(\widetilde{r}_{i j}^{1}\right)-1\right|\right\}}{\max _{1 \leq m, 1 \leq j \leq n}\left\{\left|R P_{\widetilde{r}_{i j}^{2}}\left(\widetilde{r}_{i j}^{1}\right)-1\right|\right\}-\min _{1 \leq i \leq m, 1 \leq j \leq n}\left\{\left|R P_{\widetilde{r}_{i j}^{2}}\left(\widetilde{r}_{i j}^{1}\right)-1\right|\right\}} .
$$

Definition 9 [20]. For two picture fuzzy evaluation matrices $\widetilde{R}^{1}=\left(\widetilde{r}_{i j}^{1}\right)_{m \times n}=\left(\mu_{i j}^{1}, \eta_{i j}^{1}, v_{i j}^{1}\right)_{m \times n}$ and $\widetilde{R}^{2}=\left(\widetilde{r}_{i j}^{2}\right)_{m \times n}=\left(\mu_{i j^{\prime}}^{2}, \eta_{i j}^{2}, v_{i j}^{2}\right)_{m \times n}$, the similarity degree of $\widetilde{R}^{1}$ and $\widetilde{R}^{2}$ is defined as:

$$
S D\left(\widetilde{R}^{1}, \widetilde{R}^{2}\right)=\frac{1}{2}\left(\left(1-R P_{\widetilde{R}^{2}}\left(\widetilde{R}^{1}\right)\right)+\left(1-R P_{\widetilde{R}^{1}}\left(\widetilde{R}^{2}\right)\right)\right)
$$

Let $\left\{\widetilde{R}^{1}, \widetilde{R}^{2}, \ldots, \widetilde{R}^{q}\right\}$ be a set of picture fuzzy evaluation matrices. The similarity degree of an evaluation matrix $\widetilde{R}^{k}$ to all of the others can be defined as [19]:

$$
S D\left(\widetilde{R}^{k}\right)=\frac{\sum_{i=1, i \neq k}^{q} S D\left(\widetilde{R}^{k}, \widetilde{R}^{i}\right)}{q-1}(k=1,2, \ldots q) .
$$

Definition 10 [20]. Given two picture fuzzy evaluation matrices $\widetilde{R}^{k}=\left(\widetilde{r}_{i j}^{k}\right)_{m \times n}=\left(\mu_{i j}^{k}, \eta_{i j}^{k}, v_{i j}^{k}\right)_{m \times n}$ and $\widetilde{R}=\left(\widetilde{r}_{i j}\right)_{m \times n}=\left(\mu_{i j}, \eta_{i j}, v_{i j}\right)_{m \times n}$, the consensus degree of $\widetilde{R}^{k}$ to $\widetilde{R}$ is computed by

$$
C D\left(\widetilde{R}^{k}\right)=\frac{1}{m n} \sum_{i=1}^{n} \sum_{j=1}^{m} \frac{\left|R P_{\widetilde{r}_{i j}}\left(\widetilde{r}_{i j}^{k}\right)-1\right|-\min _{1 \leq i \leq m, 1 \leq j \leq n}\left\{\left|R P_{\widetilde{r}_{i j}}\left(\widetilde{r}_{i j}^{k}\right)-1\right|\right\}}{\max _{1 \leq i \leq n}\left\{\left|R P_{\widetilde{r}_{i j}}\left(\widetilde{r}_{i j}^{k}\right)-1\right|\right\}-\min _{1 \leq i \leq m, 1 \leq j \leq n}\left\{\left|R P_{\widetilde{r}_{i j}}\left(\widetilde{r}_{i j}^{k}\right)-1\right|\right\}} .
$$

\subsection{Defuzzification of Picture Fuzzy Numbers (PFNs)}

Suppose that $\widetilde{a}=(\mu, \eta, v)$ is a PFN, where $\pi=1-\mu-\eta-v$. A defuzzification method to obtain a crisp value of the PFN is introduced as follows [21,32]:

Step 1: Distribute the neutral degree to the positive degree and negative degree as follows:

$$
\begin{aligned}
& \mu^{\prime}=\mu+\frac{\eta}{2}, \\
& v^{\prime}=v+\frac{\eta}{2} .
\end{aligned}
$$


Step 2: Calculate the defuzzification value $y$ by

$$
y=\mu^{\prime}+\frac{1+\mu^{\prime}-v^{\prime}}{2} \pi
$$

\section{Fuzzy Petri Nets (FPNs) and Fuzzy Production Rules (FPRs)}

\subsection{Fuzzy Petri Nets}

To present the FPRs of a rule-based decision-making system, the concept of FPNs, which is a potential modeling technique for knowledge representation and reasoning, was first proposed by Looney [33]. Later, Chen et al. [34] defined an FPN model to perform knowledge reasoning automatically.

Definition 11 [34]. An FPN is denoted as an 8-tuple:

$$
F P N=(\mathrm{P}, \mathrm{T}, \mathrm{D}, \mathrm{I}, \mathrm{O}, f, \alpha, \beta)
$$

where:

(1) $P=\left\{p_{1}, p_{2}, \ldots, p_{m}\right\}$ is a finite set of places;

(2) $T=\left\{t_{1}, t_{2}, \ldots, t_{n}\right\}$ is a finite set of transitions;

(3) $D=\left\{d_{1}, d_{2}, \ldots, d_{m}\right\}$ denotes a finite set of propositions with $P \cap T \cap D=\varnothing$ and $|P|=|D|$;

(4) $I: T \rightarrow P^{\infty}$ denotes the input function, a mapping from transitions to bags of places;

(5) $O: T \rightarrow P^{\infty}$ denotes the output function, a mapping from transitions to bags of places;

(6) $f: T \rightarrow[0,1]$ denotes an association function, a mapping from transitions to certainty factors between 0 and 1 ;

(7) $\quad \alpha: P \rightarrow[0,1]$ denotes an association function, a mapping from places to truth degrees between 0 and 1 ;

(8) $\quad \beta: P \rightarrow D$ denotes an association function, a bijective mapping from places to propositions.

\subsection{Fuzzy Production Rules}

In many decision support systems, vague and unprecise expert knowledge is hard to capture and store. For representing knowledge properly, FPRs, which are represented in the form of fuzzy if-then rules, have been comprehensively used to represent rule-based knowledge. In FPRs, the antecedent and the consequent of each rule contain the fuzzy concepts expressed by fuzzy sets. The FPR is called a composite FPR if it contains either AND or OR connectors [35,36].

Later, the weight parameter is been assigned into FPRs to define the weighted FPRs (WFPRs) [35], enhancing the power of FPRs to present fuzzy data. Let $R=\left\{R_{1}, R_{2}, \ldots, R_{n}\right\}$ be a set of WFPRs. The $i$ th rule can be defined as follows:

$$
R_{i}: \operatorname{IF} a \text { THEN } c(C F=u), T h, w,
$$

where:

(1) $a=\left\{a_{1}, a_{2}, \ldots, a_{m}\right\}$ is the antecedent portion of the rule $R_{i}$, comprising one or more propositions with some fuzzy variables connected by either "AND" or "OR";

(2) $\quad c=\left\{c_{1}, c_{2}, \ldots, c_{m}\right\}$ is the consequent portion of the rule $R_{i}$, comprising one or more propositions with some fuzzy variables connected by either "AND" or "OR";

(3) $u(u \in[0,1])$ is the certainty factor of the rule $R_{i}$, representing the belief strength of the rule;

(4) $T h=\left\{\lambda_{1}, \lambda_{2}, \ldots, \lambda_{m}\right\}$ is a set of threshold values specified for each of the propositions in the antecedent; 
(5) $w=\left\{w_{1}, w_{2}, \ldots, w_{m}\right\}$ is a set of weights assigned to the propositions in the antecedent portion.

In general, the WFPRs can be divided into five types as follows [37-39]:

Type 1. A simple WFPR: $R$ : IF $a$ THEN $c(u ; \lambda ; w)$;

Type 2. A composite weighted fuzzy conjunctive rule in the antecedent: $R$ : IF $a_{1}$ AND $a_{2}$ AND $\ldots$ AND $a_{m}$ THEN $c\left(u ; \lambda_{1}, \lambda_{2}, \ldots, \lambda_{m} ; w_{1}, w_{2}, \ldots, w_{m}\right)$;

Type 3. A composite weighted fuzzy conjunctive rule in the consequent: $R$ : IF $a$ THEN $c_{1}$ AND $c_{2}$ AND ... AND $c_{m}(u ; \lambda ; w)$;

Type 4. A composite weighted fuzzy disjunctive rule in the antecedent: $R$ : IF $a_{1}$ OR $a_{2}$ OR .. OR $a_{m}$ THEN $c\left(u ; \lambda_{1}, \lambda_{2}, \ldots, \lambda_{m} ; w_{1}, w_{2}, \ldots, w_{m}\right)$;

Type 5. A composite weighted fuzzy disjunctive rule in the consequent: $R$ : IF $a \operatorname{THEN} c_{1}$ OR $c_{2}$ OR $\ldots$ OR $c_{m}(u ; \lambda ; w)$.

In many real situations, the rules of Type 4 and Type 5, which can be transferred into several rules of Type 1, are not allowed to be appeared in an expert system.

\section{Picture Fuzzy Petri Nets (PFPNs)}

\subsection{Definition of PFPNs}

With the increasing of complexity of rule-based systems, a new FPN model based on PFSs, namely picture fuzzy Petri nets (PFPNs), is proposed for knowledge representation and reasoning in this section.

Definition 12. Let $\widetilde{\Psi}$ be the set of all PFNs defined in the universe X. A PFPN definition can be described as follows:

$$
P F P N=(P, T, D, I, O, \widetilde{M}, T h, \widetilde{U}, L W, G W),
$$

where:

(1) $P, T$, and $D$ are denoted as in Definition 11;

(2) I: $P \times T \rightarrow\{0,1\}$ is an $m \times n$ input incidence matrix defining the directed arcs from places to transitions. If there exists an arc from input place $p_{i}$ to transition $t_{j}$, then $I_{i j}=1$, otherwise $I_{i j}=0$, for $i=1,2, \ldots, m, j=1,2, \ldots, n$;

(3) $O:[T \times P]^{T} \rightarrow\{0,1\}$ is an $m \times n$ output incidence matrix defining the directed arcs from transitions to places. If there exists an arc from transition $t_{j}$ to output places $p_{i}$, then $O_{i j}=1$, otherwise $O_{i j}=0$, for $i=1,2, \ldots, m, j=1,2, \ldots, n$;

(4) $\tilde{M}: P \rightarrow \widetilde{\Psi}$ is a marking vector $\widetilde{M}=\left(\widetilde{\alpha}_{1}, \widetilde{\alpha}_{2}, \ldots, \widetilde{\alpha}_{m}\right)^{T}$, which indicates a mapping from places to PFNs and denotes the truth degree of the place $p_{i}$. The starting vector is denoted by $\widetilde{M}_{0}$.

(5) $\widetilde{T h}: P \rightarrow \widetilde{\Psi}$ denotes a vector $\widetilde{T h}=\left(\widetilde{\lambda}_{1}, \widetilde{\lambda}_{2}, \ldots, \widetilde{\lambda}_{m}\right)^{T}$. The function assigns a threshold value $\widetilde{\lambda}_{i}$ expressed by a PFN to each input place of a transition;

(6) $\widetilde{U}: T \rightarrow \widetilde{\Psi}$ denotes a vector $\widetilde{U}=\left(\widetilde{\mu}_{1}, \widetilde{\mu}_{2}, \ldots \widetilde{\mu}_{n}\right)$. The element $\widetilde{\mu}_{i}$ is expressed by a PFN, which denotes the certainty factor of the transition $t_{i}$;

(7) $L W: P \rightarrow[0,1]$ is a set of local weights of places, which can be expressed as a vector $L W=$ $\left(l w_{1}, l w_{2}, \ldots, l w_{m}\right)^{T}$. Here, $l w_{i}$ is a real value between 0 and 1 , representing the relative importance of the input place $p_{i}$ contributing to the transition $t_{j}$;

(8) $G W: T \rightarrow[0,1]$ is a set of global weights of transitions, which can be expressed as a vector $G W=\left(g w_{1}, g w_{2}, \ldots, g w_{n}\right)$. The element $g w_{j} \in[0,1]$ is the global weight which reflects how much a transition impacts its output places. 


\subsection{PFPN Representations of Weight Picture Fuzzy Production Rules (WPFPRs)}

In this article, a decision support system is supposed to be modelled by the weight picture fuzzy production rules (WPFPRs). In order to map WPFPRs into PFPNs, we define WPFPRs in the following new forms:

Type 1. A simple WPFPRs $R$ : IF $a \operatorname{THEN} c(\widetilde{\lambda} ; l w ; \widetilde{\mu} ; g w)$;

Type 2. A composite-weighted picture fuzzy conjunctive rule in the antecedent $R$ : IF $a_{1}$ AND $a_{2}$ AND $\ldots$ AND $a_{m} \operatorname{THEN} c\left(\widetilde{\lambda}_{1}, \widetilde{\lambda}_{2}, \ldots \widetilde{\lambda}_{m} ; l w_{1}, l w_{2}, \ldots, l w_{m} ; \tilde{\mu} ; g w\right)$;

Type 3. A composite weighted picture fuzzy conjunctive rule in the consequent $R$ : IF $a$ THEN $c_{1}$ AND $c_{2} \operatorname{AND} \ldots \operatorname{AND} c_{m}(\widetilde{\lambda} ; l w ; \tilde{\mu} ; g w)$.

The above three types of WPFPRs can be represented by PFPNs, as shown in Figures 1-3, respectively.

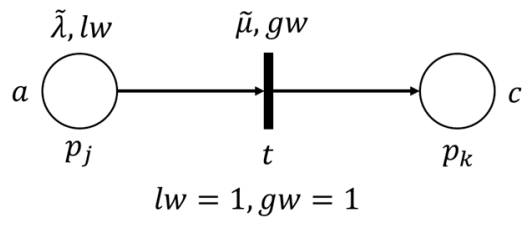

Figure 1. Picture fuzzy Petri net (PFPN) representation of Type 1 rule.

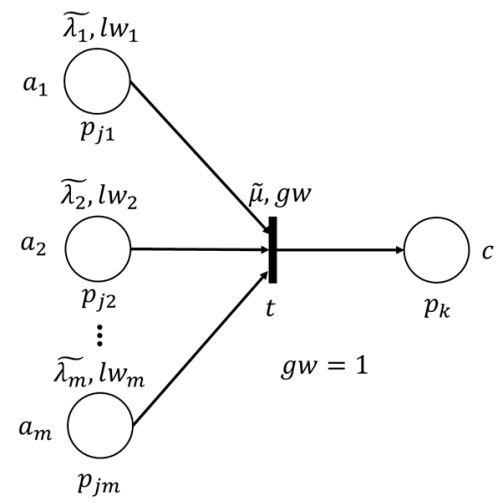

Figure 2. PFPN representation of Type 2 rule.

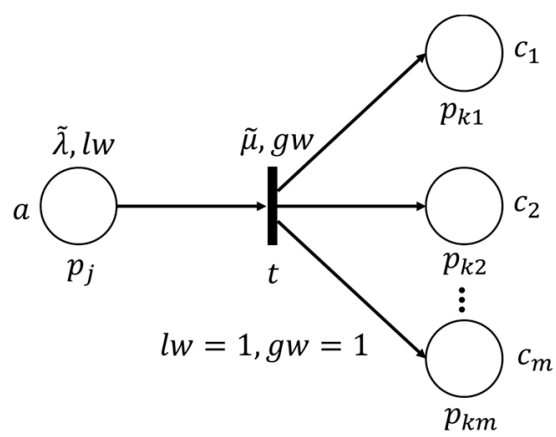

Figure 3. PFPN representation of Type 3 rule.

\subsection{Knowledge Acquisition}

In this part, an approach to determine the knowledge parameters of PFPNs, i.e., global weight $(G W)$, local weight $(L W)$, threshold value $(\widetilde{T h})$, and certainty factor $(\widetilde{U})$, is introduced. Particularly, the conflict and inconsistency among expert evaluations and the vagueness in the knowledge parameters can be considered in the knowledge acquisition process. 
Suppose that there are $N$ WPFPRs $\left\{R_{1}, R_{2}, \ldots, R_{N}\right\}$ to be evaluated by $K$ experts $\left\{T M_{1}, T M_{2}, \ldots, T M_{K}\right\}$ with respect to $L$ antecedent propositions $\left\{P_{1}, P_{2}, \ldots, P_{L}\right\}$. For a WPFPR, local weight and threshold value are related to the antecedent propositions; global weight and certainty factor are related to the WPFPRs. To simplify the discussion, only the process of determining the local weight $(L W)$ is explained here.

For the local weight, suppose that an expert $T M_{k}$ provides the assessment of $R_{n}$ with respect to $P_{l}$ by a PFN denoted as $\widetilde{\gamma}^{k}\left(P_{l}\right)=\left(\mu_{l}^{k}, \eta_{l}^{k}, v_{l}^{k}\right)$. Then, a picture fuzzy evaluation vector for the $N$ WPFPRs with respect to $L$ antecedent propositions can be formed as $\widetilde{X}^{k}=\left(\widetilde{\gamma}^{k}\left(P_{1}\right), \widetilde{\gamma}^{k}\left(P_{2}\right), \ldots, \widetilde{\gamma}^{k}\left(P_{L}\right)\right)^{T}$. The steps to determine the $L W$ are described as follows:

Step 1: Determine the weights of experts.

Let $\widetilde{X}^{k}(k=1,2, \ldots K)$ be the picture fuzzy evaluation vectors of local weights provided by all the experts. The similarity degree of $\widetilde{X}^{k}$ to all the others can be calculated by:

$$
S D\left(\widetilde{X}^{k}\right)=\frac{\sum_{i=1, i \neq k}^{K} S D\left(\widetilde{X}^{k}, \widetilde{X}^{i}\right)}{K-1} .
$$

Then, the weight of each expert can be obtained as follows [20]:

$$
\omega_{k}=\left\{\begin{array}{c}
F\left(\frac{S D\left(\widetilde{X}_{o(1)}\right)}{T}\right), k=1, \\
F\left(\frac{\sum_{i=1}^{k} S D\left(\widetilde{X}_{o(i)}\right)}{T}\right)-F\left(\frac{\sum_{i=1}^{k-1} S D\left(\widetilde{X}_{o(i)}\right)}{T}\right), k=2,3, \ldots, K,
\end{array}\right.
$$

where $\omega_{k}$ satisfies $\sum_{k=1}^{K} \omega_{k}=1, T=\sum_{k=1}^{K} S D\left(\widetilde{X}^{k}\right)$ is the sum of all the similarity degrees, and $\left\{S D\left(\widetilde{X}_{o(1)}\right), S D\left(\widetilde{X}_{o(2)}\right), \ldots, S D\left(\widetilde{X}_{o(K)}\right)\right\}$ is a decreasing ranking value of $\left\{S D\left(\widetilde{X}^{1}\right), S D\left(\widetilde{X}^{2}\right), \ldots, S D\left(\widetilde{X}^{K}\right)\right\} . \quad F(a)=a^{\lambda}$ is a function to avoid the situation where the generated weights differ greatly from each other.

Step 2: Aggregate all the picture fuzzy evaluation vectors.

The picture fuzzy evaluation vectors $\widetilde{X}^{k}(k=1,2, \ldots K)$ can be aggregated to obtain a collective picture fuzzy evaluation vector $\widetilde{X}=\left(\widetilde{\gamma}\left(P_{1}\right), \widetilde{\gamma}\left(P_{2}\right), \ldots, \widetilde{\gamma}\left(P_{L}\right)\right)^{T}$ with the PFWG operator. For each element of $\widetilde{X}$, the $\widetilde{\gamma}\left(P_{l}\right)$ will be computed by:

$$
\begin{aligned}
\widetilde{\gamma}\left(P_{l}\right)= & \operatorname{PFWG}\left(\widetilde{\gamma}^{1}\left(P_{l}\right), \widetilde{\gamma}^{2}\left(P_{l}\right), \ldots, \widetilde{\gamma}^{K}\left(P_{l}\right)\right) \\
& =\left\langle\prod_{j=1}^{K}\left(\mu_{l}^{j}+\eta_{l}^{j}\right)^{\omega_{j}}-\prod_{j=1}^{K}\left(\eta_{l}^{j}\right)^{\omega_{j}}, \prod_{j=1}^{K}\left(\eta_{l}^{j}\right)^{\omega_{j}}, 1-\prod_{j=1}^{K}\left(1-v_{l}^{j}\right)^{\omega_{j}}\right\rangle
\end{aligned}
$$

Step 3: Consensus checking and improving.

Once the collective picture fuzzy evaluation vector $\widetilde{X}=\left(\widetilde{\gamma}\left(P_{1}\right), \widetilde{\gamma}\left(P_{2}\right), \ldots, \widetilde{\gamma}\left(P_{L}\right)\right)^{T}$ is gained, the consensus degree of the vector $\widetilde{X}^{k}=\left(\widetilde{\gamma}^{k}\left(P_{1}\right), \widetilde{\gamma}^{k}\left(P_{2}\right), \ldots, \widetilde{\gamma}^{k}\left(P_{L}\right)\right)^{T}$ to $\widetilde{X}$ can be computed as below:

$$
C D\left(\widetilde{X}^{k}\right)=\frac{1}{L} \sum_{l=1}^{L} \frac{\left|R P_{\widetilde{\gamma}\left(P_{l}\right)}\left(\widetilde{\gamma}^{k}\left(P_{l}\right)\right)-1\right|-\min _{1 \leq l \leq L}\left\{\left|R P_{\widetilde{\gamma}\left(P_{l}\right)}\left(\widetilde{\gamma}^{k}\left(P_{l}\right)\right)-1\right|\right\}-\min _{1 \leq l \leq L}\left\{\left|R P_{\widetilde{\gamma}\left(P_{l}\right)}\left(\widetilde{\gamma}^{k}\left(P_{l}\right)\right)-1\right|\right\}}{\left.\left(\widetilde{\gamma}^{k}\left(P_{l}\right)\right)-1 \mid\right\}} .
$$

In general, if $C D\left(\widetilde{X}^{k}\right)=0$, the consensus of $\widetilde{X}^{k}$ to $\widetilde{X}$ is extremely high. If $C D\left(\widetilde{X}^{k}\right) \leq \theta$, the consensus of $\widetilde{X}^{k}$ to $\widetilde{X}$ is acceptable, where $\theta$ is the threshold value provided by each expert, i.e., 
$\theta=\min \left\{\theta_{1}, \theta_{2}, \ldots, \theta_{K}\right\}$. However, if $C D\left(\widetilde{X}^{k}\right)>\theta$, which means that the consensus of $\widetilde{X}^{k}$ to $\widetilde{X}$ is not acceptable, the picture fuzzy evaluation vector $\widetilde{X}^{k}$ should be modified according to the following rules:

(1) If $\widetilde{\gamma}^{k}\left(P_{l}\right)<\widetilde{\gamma}\left(P_{l}\right)$, then expert $T M_{k}$ should increase his/her assessment of local weight with respect to $P_{l}$;

(2) If $\widetilde{\gamma}^{k}\left(P_{l}\right)>\widetilde{\gamma}\left(P_{l}\right)$, then expert $T M_{k}$ should decrease his/her assessment of local weight with respect to $P_{l}$;

(3) If $\widetilde{\gamma}^{k}\left(P_{l}\right)=\widetilde{\gamma}\left(P_{l}\right)$, then expert $T M_{k}$ will not revise his/her assessment of local weight with respect to $P_{l}$.

Step 4: Determine the local weights of antecedent propositions.

After consensus checking and improving, the updated collective picture fuzzy evaluation vector $\widetilde{X}^{\prime}$ can be obtained. Then, it should be defuzzified into a crisp evaluation vector $X=\left(x_{1}, x_{2}, \ldots, x_{L}\right)$ by using the defuzzification method given in Section 2.2. Next, the crisp evaluation vector $X$ needs to be normalized by

$$
l w_{h}=\frac{x_{h}}{\sum_{i=1}^{H} x_{i}}, h=1,2, \ldots, H,
$$

where $l w_{h}(h=1,2, \ldots, H)$ are the local weights with respect to each WPFPR's antecedent propositions.

The knowledge parameters of global weight $(G W)$, threshold value $(\widetilde{T h})$, and certainty factor $(\widetilde{U})$ can be acquired in the same way. For the $G W$, its collective picture fuzzy evaluation vector needs to be defuzzified and normalized as well, while the $\widetilde{T h}$ and the $\widetilde{U}$ can be directly determined from their collective picture fuzzy evaluation vectors.

\subsection{Execution Rules of PFPNs}

A marked PFPN is a PFPN with some places containing tokens. Let $I(t)=\left\{p_{I 1}, p_{I 2}, \ldots, p_{I m}\right\}$ be input places of transition $t$. The threshold values $\left\{\lambda_{I 1}, \lambda_{I 1}, \ldots, \lambda_{I m}\right\}$ and local weights $\left\{l w_{I 1}, l w_{I 2}, \ldots, l w_{I m}\right\}$ are assigned to input places, while the certainty factor and global weight of a transition $t$ are expressed by $\widetilde{\mu}_{t}$ and $g w_{t}$, respectively. Next, the enabling and firing rules of PFPNs are introduced.

(1) Enabling Rule: The transition $t$ is enabled if:

$$
\widetilde{\alpha}\left(p_{I j}\right) \geq \lambda_{I j}, j=1,2, \ldots, m,
$$

where $\widetilde{\alpha}\left(p_{I j}\right)$ is the truth value of place $p_{I j}$ and $\widetilde{\lambda}_{I j}$ is the threshold value of $p_{I j}$.

(2) Firing Rule: Let $p_{O}$ be an output place of transition $t$. When transition $t$ is fired, the truth values of its input places will be copied and deposited into its output places. If $p_{O}$ has only one input transition, the truth value of $p_{O}$ is computed by

$$
\widetilde{\alpha}\left(p_{O}\right)=\operatorname{PFWG}\left(\widetilde{\alpha}\left(p_{I 1}\right), \widetilde{\alpha}\left(p_{I 2}\right), \ldots, \widetilde{\alpha}\left(p_{I m}\right)\right) \otimes \widetilde{\mu}_{i}
$$

where the PFWG weight vector is $w=\left(l w_{1}, l w_{2}, \ldots, l w_{m}\right)$.

If $p_{O}$ has more than one fired input transitions $t_{i}(i=1,2 \ldots, n, i>1)$, then the truth value of $p_{O}$ is defined by

$$
\widetilde{\alpha}\left(p_{O}\right)=\operatorname{PFWG}\left(\widetilde{\alpha}\left(p_{O 1}\right), \widetilde{\alpha}\left(p_{O 2}\right), \ldots, \widetilde{\alpha}\left(p_{O n}\right)\right),
$$

where the PFWG weight vector is $w=\left(g w_{1}, g w_{2}, \ldots, g w_{n}\right)$ and $\widetilde{\alpha}\left(p_{O i}\right)$ is the truth value determined by the $i$ th input transition. 


\subsection{Reasoning Algorithm Based on PFPNs}

In this section, based on the basic matrix operators introduced in [16], a concurrent inference algorithm of PFPNs is proposed.

Input: $I$ and $O$ are $m \times n$-dimensional matrices, $\widetilde{U}$ and $G W$ are two $n$-dimensional vectors, and $L W, T h$, and $\widetilde{M}_{0}$ are $m$-dimensional vectors.

Output: $\widetilde{M}_{k}$ is an $m$-dimensional vector, signifying the final truth degree of all the propositions.

Step 1: Let $k=1$. The parameter $k$ denotes the time of iterations.

Step 2: Calculate the enabled place vector $D^{(k)}$. $D^{(k)}$ can be obtained by comparing the truth values of the places with their threshold values, which indicates the enabled input places of transitions.

$$
D^{(k)}=\widetilde{M}_{k-1} \triangleright \widetilde{T h} .
$$

Step 3: Calculate the token value vector of input places $\widetilde{\Gamma}^{(k)}$ with respect to the local weight of each input place. If $D^{(k)}$ is a nonzero matrix, $\widetilde{\Gamma}^{(k)}$ can be computed by Equation (26); otherwise, go to Step 7.

$$
\widetilde{\Gamma}^{(k)}=\operatorname{PFWG}\left((I \circ W)^{T}, \widetilde{M}_{k-1}\right),
$$

where $W=[L W, L W, \ldots, L W]_{m \times n}$.

Step 4: Calculate the enabled transition vector $F^{(k)}$ by Equation (27), which indicates the enabled transitions of output places.

$$
F^{(k)}=(E \times I) \triangleright\left(\left(D^{(k)}\right)^{T} \times I\right)
$$

where $E=(1)_{1 \times m}=[1,1, \ldots, 1]$.

Step 5: Calculate the output truth degree vector $\Theta^{(k)}$. If $F^{(k)}$ is a nonzero matrix, $\Theta^{(k)}$ can be calculated by Equation (28); otherwise, go to Step 7.

$$
\Theta^{(k)}=\left(F^{(k)} \circ \Gamma^{(k)}\right) \circ \widetilde{U}
$$

Step 6: Compute the new marking vector $\widetilde{M}_{k}$.

$$
\widetilde{M}_{k}=\widetilde{M}_{k-1} \oplus \operatorname{PFWG}\left(\left(O \circ W_{g w}\right), \Theta^{(k)}\right) .
$$

where $W_{g w}=[G W, G W, \ldots, G W]_{m \times n}^{T}$. If $\widetilde{M}_{k}=\widetilde{M}_{k-1}$, then go to Step 7; otherwise let $k=k+1$, go back to Step 2.

Step 7: The reasoning is over.

\section{Illustrative Example}

In this section, a realistic example regarding a gene regulatory network with activating and repressing processes [40] is provided to show the applicability and feasibility of the PFPN model.

\subsection{Implementation}

The gene regulatory network controls biological functions by regulating the gene expression levels [40]. It is meaningful to understand the complex causal relationships within a gene regulatory network in a biological system. Genes are paired into activator and repressor, and this gene pair determines the predicted target gene expression level. In what follows, the proposed PFPN model is used to model the gene regulatory network to predict changes in the expression level of the target gene. 
Let $d_{i}(i=1,2, \ldots, 9)$ be nine genes (nine propositions). The WPFPRs of the gene network are defined as follows:

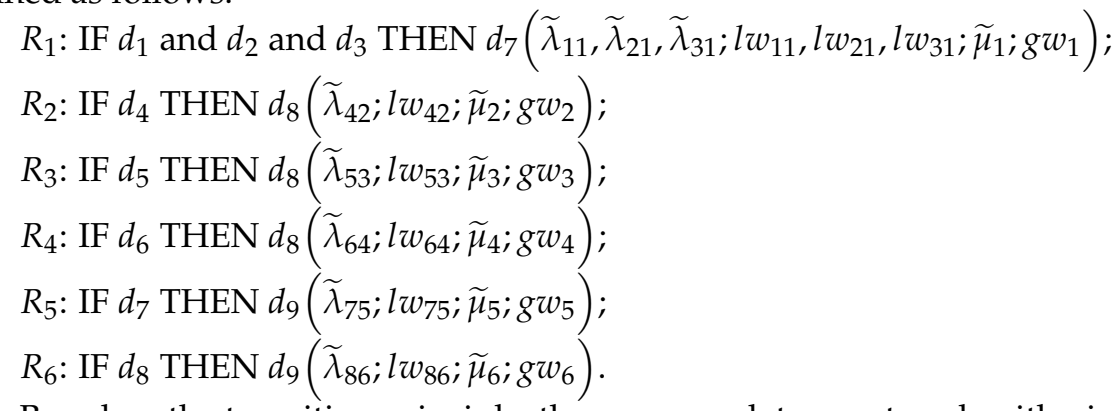

Based on the transition principle, the gene regulatory network with nine genes can be modeled by a PFPN, as shown in Figure 4. According to the gene regulatory network of a biological system, the places in the PFPN with respect to their relative propositions are presented in Table 1 . The places $p_{i}(i=1,2, \ldots, 6)$ are called starting places, the places $p_{7}$ and $p_{8}$ are called intermediate places, and the place $p_{9}$ is a terminating place.

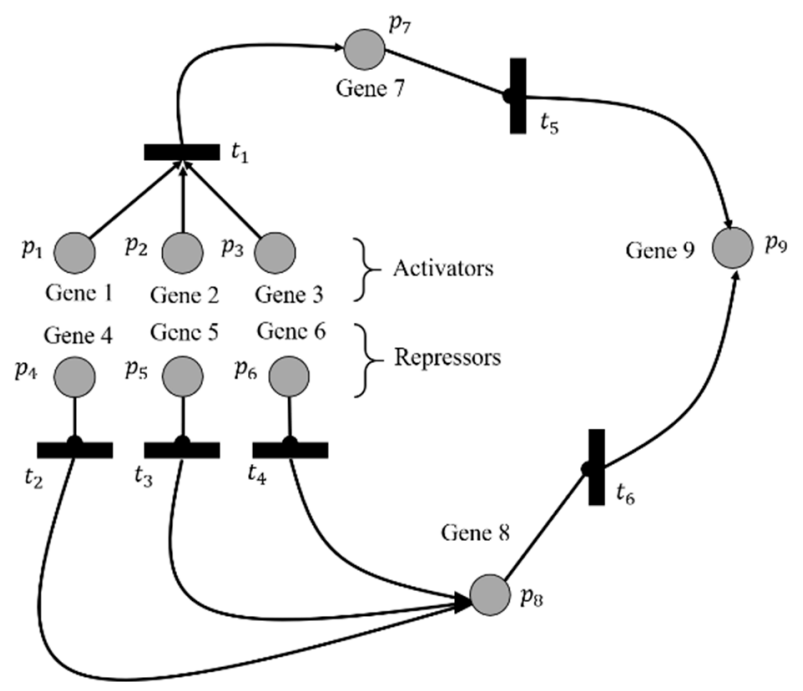

Figure 4. PFPN model for the gene network.

Table 1. Places of the PFPN model and their propositions.

\begin{tabular}{cc}
\hline Place $\left(p_{i}\right)$ & Proposition $\left(d_{i}\right)$ \\
\hline$p_{1}$ & Activator gene 1 that increases gene 7 expression \\
$p_{2}$ & Activator gene 2 that increases gene 7 expression \\
$p_{3}$ & Activator gene 3 that increases gene 7 expression \\
$p_{4}$ & Repressor gene 4 that decreases gene 8 expression \\
$p_{5}$ & Repressor gene 5 that decreases gene 8 expression \\
$p_{6}$ & Repressor gene 6 that decreases gene 8 expression \\
$p_{7}$ & Repressor gene 7 that decreases gene 9 expression \\
$p_{8}$ & Repressor gene 8 that decreases gene 9 expression \\
$p_{9}$ & Target gene 9 \\
\hline
\end{tabular}

To determine the knowledge parameters of local weights, global weights, threshold values, and certainty factors, five experts $\left\{T M_{1}, T M_{2}, T M_{3}, T M_{4}, T M_{5}\right\}$ are invited to provide their judgements with respect to the six WPFPRs. As it is not easy for the experts to express unprecise knowledge information, a linguistic term set $S$ is utilized to evaluate the above knowledge parameters: $S=\left\{s_{0}=\right.$ Very Low,$s_{1}=$ Low,$s_{2}=$ Moderate,$s_{3}=$ High,$s_{4}=$ Very High $\}$. All the five linguistic terms can be approximated by PFNs, as outlined in Table 2. 
Table 2. Linguistic term set defined by picture fuzzy numbers (PFNs).

\begin{tabular}{cc}
\hline Linguistic Term & Picture Fuzzy Number \\
\hline Very Low & $(0,0,0.9)$ \\
Low & $(0,0.5,0.4)$ \\
Moderate & $(0,0.9,0)$ \\
High & $(0.5,0.4,0)$ \\
Very High & $(0.9,0,0)$ \\
\hline
\end{tabular}

For the gene regulatory network, the initial picture fuzzy evaluation vectors of local weights, global weights, threshold values, and certainty factors, provided by the five experts, are shown in Tables 3-6, respectively. Then, the knowledge parameters of the six WPFPRs are acquired according to the proposed knowledge acquisition approach.

First, the weight of each expert is obtained using Equations (17) and (18). Second, the picture fuzzy evaluation vectors of experts are synthesized into a collective picture fuzzy evaluation vector using Equation (19). Next, the consensus degrees of the experts can be checked according to Equation (20) and improved until they are less than or equal to the consensus degree threshold value $\theta(\theta=0.5)$. Consequently, the computation results for the four knowledge parameters are displayed in Tables 3-6, respectively. Note that the collective picture fuzzy evaluation vectors of local weights and global weights should be defuzzified and normalized, as presented in the last columns of Tables 3 and 4 . 
Table 3. Local weights on six weight picture fuzzy production rules (WPFPRs) by five experts.

\begin{tabular}{|c|c|c|c|c|c|c|c|c|}
\hline \multirow{2}{*}{ WPFPRs } & \multirow{2}{*}{ Local Weight } & \multicolumn{5}{|c|}{ Initial Picture Fuzzy Evaluation Vectors } & \multirow{2}{*}{$\begin{array}{l}\text { Collective Picture } \\
\text { Fuzzy Evaluations }\end{array}$} & \multirow{2}{*}{$\begin{array}{l}\text { Defuzzification and } \\
\text { Normalization }\end{array}$} \\
\hline & & $\mathrm{TM}_{1}$ & $\mathrm{TM}_{2}$ & $\mathrm{TM}_{3}$ & $\mathrm{TM}_{4}$ & $\mathrm{TM}_{5}$ & & \\
\hline & $l w_{11}$ & $(0.9,0,0)$ & $(0.8,0.05,0.05)$ & $(0.7,0.1,0.1)$ & $(0.8,0.05,0.05)$ & $(0.8,0,0.1)$ & $(0.862,0,0.038)$ & 0.468 \\
\hline \multirow[t]{2}{*}{1} & $l w_{21}$ & $(0.5,0.1,0.3)$ & $(0.4,0.2,0.3)$ & $(0.5,0.2,0.2)$ & $(0.6,0.2,0.1)$ & $(0.5,0.1,0.3)$ & $(0.534,0,0.362)$ & 0.292 \\
\hline & $l w_{31}$ & $(0.3,0.4,0.2)$ & $(0.3,0.4,0.2)$ & $(0.2,0.4,0.3)$ & $(0.3,0.3,0.3)$ & $(0.2,0.2,0.5)$ & $(0.304,0.27,0.324)$ & 0.240 \\
\hline 2 & $l w_{42}$ & & & & & & & 1 \\
\hline 3 & $l w_{53}$ & & & & & & & 1 \\
\hline 4 & $l w_{64}$ & & & & & & & 1 \\
\hline 5 & $l w_{75}$ & & & & & & & 1 \\
\hline 6 & $l w_{86}$ & & & & & & & 1 \\
\hline \multicolumn{2}{|c|}{ Similarity degree } & 0.625 & 0.544 & 0.635 & 0.540 & 0.551 & & \\
\hline \multicolumn{2}{|c|}{ Expert weight } & 0.216 & 0.187 & 0.223 & 0.185 & 0.190 & & \\
\hline \multicolumn{2}{|c|}{ Consensus degree } & 0.357 & 0.397 & 0.378 & 0.335 & 0.385 & & \\
\hline
\end{tabular}

Table 4. Global weights on six WPFPRs by five experts.

\begin{tabular}{|c|c|c|c|c|c|c|c|c|}
\hline \multirow{2}{*}{ WPFPRs } & \multirow{2}{*}{$\begin{array}{c}\text { Global } \\
\text { Weights }\end{array}$} & \multicolumn{5}{|c|}{ Initial Picture Fuzzy Evaluation Vectors } & \multirow{2}{*}{$\begin{array}{l}\text { Collective Picture } \\
\text { Fuzzy Evaluations }\end{array}$} & \multirow{2}{*}{$\begin{array}{l}\text { Defuzzification and } \\
\text { Normalization }\end{array}$} \\
\hline & & $\mathrm{TM}_{1}$ & $\mathrm{TM}_{2}$ & $\mathrm{TM}_{3}$ & $\mathrm{TM}_{4}$ & $\mathrm{TM}_{5}$ & & \\
\hline 1 & $g w_{1}$ & & & & & & & 1 \\
\hline 2 & $g w_{2}$ & $(0.7,0.1,0.1)$ & $(0.5,0.1,0.3)$ & $(0.5,0.2,0.2)$ & $(0.6,0.2,0.1)$ & $(0.2,0.4,0.3)$ & $(0.518,0.175,0.206)$ & 0.297 \\
\hline 3 & $g w_{3}$ & $(0.7,0.1,0.1)$ & $(0.6,0.1,0.2)$ & $(0.6,0.1,0.2)$ & $(0.6,0.1,0.2)$ & $(0.6,0.1,0.2)$ & $(0.556,0.1,0.243)$ & 0.303 \\
\hline 4 & $\begin{array}{l}g w_{3} \\
g w_{4}\end{array}$ & $(0.9,0,0)$ & $(0.7,0.1,0.1)$ & $(0.5,0.3,0.1)$ & $(0.3,0.3,0.3)$ & $(0.6,0.2,0.1)$ & $(0.829,0,0.07)$ & 0.4 \\
\hline 5 & $\begin{array}{l}g w_{4} \\
g w_{5}\end{array}$ & $(0.6,0.1,0.2)$ & $(0.6,0.1,0.2)$ & $(0.3,0.5,0.1)$ & $(0.7,0.1,0.1)$ & $(0.6,0.2,0.1)$ & $(0.725,0.103,0.072)$ & 0.602 \\
\hline 6 & $g w_{6}$ & $(0.3,0.4,0.2)$ & $(0.4,0.2,0.3)$ & $(0.5,0.3,0.1)$ & $(0.3,0.4,0.2)$ & $(0.3,0.4,0.2)$ & $(0.352,0.32,0.228)$ & 0.398 \\
\hline \multicolumn{2}{|c|}{ Similarity degree } & 0.556 & 0.588 & 0.581 & 0.599 & 0.591 & & \\
\hline \multicolumn{2}{|c|}{ Expert weight } & 0.189 & 0.201 & 0.198 & 0.209 & 0.203 & & \\
\hline \multicolumn{2}{|c|}{ Consensus degree } & 0.447 & 0.3598 & 0.459 & 0.345 & 0.36 & & \\
\hline
\end{tabular}


Table 5. Certain factors on six WPFPRs by five experts.

\begin{tabular}{|c|c|c|c|c|c|c|c|}
\hline \multirow{2}{*}{ WPFPRs } & \multirow{2}{*}{ Certainty Factors } & \multicolumn{5}{|c|}{ Initial Picture Fuzzy Evaluation Vectors } & \multirow{2}{*}{$\begin{array}{l}\text { Collective Picture } \\
\text { Fuzzy Evaluations }\end{array}$} \\
\hline & & $\mathrm{TM}_{1}$ & $\mathrm{TM}_{2}$ & $\mathrm{TM}_{3}$ & $\mathrm{TM}_{4}$ & $\mathrm{TM}_{5}$ & \\
\hline 1 & $\widetilde{\mu}_{1}$ & $(0.7,0.3,0)$ & $(0.96,0,0)$ & $(0.99,0,0)$ & $(0.98,0,0)$ & $(0.9,0,0)$ & $(0.966,0,0)$ \\
\hline 2 & $\widetilde{\mu}_{2}$ & $(0.96,0,0)$ & $(0.8,0.1,0.1)$ & $(0.99,0,0)$ & $(0.98,0,0)$ & $(0.9,0,0)$ & $(0.946,0,0.02)$ \\
\hline 3 & $\widetilde{\mu}_{3}$ & $(0.9,0,0)$ & $(0.9,0,0)$ & $(0.7,0.3,0)$ & $(0.85,0,0)$ & $(0.9,0,0)$ & $(0.907,0,0)$ \\
\hline 4 & $\widetilde{\mu}_{4}$ & $(0.99,0,0)$ & $(0.99,0,0)$ & $(0.99,0,0)$ & $(0.98,0,0)$ & $(0.99,0,0)$ & $(0.988,0,0)$ \\
\hline 5 & $\widetilde{\mu}_{5}$ & $(0.99,0,0)$ & $(0.99,0,0)$ & $(0.7,0.2,0.1)$ & $(0.7,0.3,0)$ & $(0.99,0,0)$ & $(0.974,0,0.02)$ \\
\hline 6 & $\widetilde{\mu}_{6}$ & $(0.96,0,0)$ & $(0.99,0,0)$ & $(0.7,0.3,0)$ & $(0.9,0.1,0)$ & $(0.7,0.3,0)$ & $(0.988,0,0)$ \\
\hline \multicolumn{2}{|c|}{ Similarity degree } & 0.733 & 0.662 & 0.723 & 0.794 & 0.747 & \\
\hline \multicolumn{2}{|c|}{ Consensus degree } & 0.227 & 0.197 & 0.3 & 0.285 & 0.24 & \\
\hline
\end{tabular}

Table 6. Threshold values on six WPFPRs by five experts.

\begin{tabular}{|c|c|c|c|c|c|c|c|}
\hline \multirow{2}{*}{ WPFPRs } & \multirow{2}{*}{ Threshold Values } & \multicolumn{5}{|c|}{ Initial Picture Fuzzy Evaluation Vectors } & \multirow{2}{*}{$\begin{array}{l}\text { Collective Picture } \\
\text { Fuzzy Evaluations }\end{array}$} \\
\hline & & $\mathbf{T M}_{1}$ & $\mathbf{T M}_{2}$ & $\mathrm{TM}_{3}$ & $\mathbf{T M}_{4}$ & $\mathrm{TM}_{5}$ & \\
\hline & $\widetilde{\lambda}_{11}$ & $(0.7,0.1,0.1)$ & $(0.3,0.25,0.35)$ & $(0.4,0.2,0.3)$ & $(0.4,0.2,0.4)$ & $(0.1,0.3,0.6)$ & $(0.196,0.2,0.604)$ \\
\hline \multirow[t]{2}{*}{1} & $\widetilde{\sim}_{21}$ & $(0.5,0.1,0.3)$ & $(0.1,0.1,0.7)$ & $(0.3,0.1,0.5)$ & $(0.4,0.1,0.4)$ & $(0.25,0.05,0.6)$ & $(0.252,0.086,0.558)$ \\
\hline & $\widetilde{\lambda}_{31}$ & $(0.1,0.2,0.7)$ & $(0.2,0.1,0.6)$ & $(0.2,0.3,0.4)$ & $(0.1,0.3,0.5)$ & $(0.05,0.2,0.65)$ & $(0.12,0.202,0.599)$ \\
\hline 2 & $\tilde{\lambda}_{42}$ & $(0.1,0.05,0.85)$ & $(0.4,0.05,0.55)$ & $(0.1,0.1,0.7)$ & $(0.3,0.05,0.65)$ & $(0.2,0.01,0.79)$ & $(0.186,0.025,0.789)$ \\
\hline 3 & $\stackrel{\sim}{\sim}_{53}$ & $(0.2,0.1,0.6)$ & $(0.2,0.1,0.6)$ & $(0.25,0.05,0.6)$ & $(0.4,0.3,0.2)$ & $(0.2,0.1,0.6)$ & $(0.243,0.106,0.547)$ \\
\hline 4 & $\tilde{\lambda}_{64}$ & $(0.1,0.15,0.65)$ & $(0.2,0.15,0.55)$ & $(0.05,0.15,0.7)$ & $(0.5,0.25,0.15)$ & $(0.05,0.25,0.6)$ & $(0.106,0.184,0.608)$ \\
\hline 6 & $\tilde{\lambda}_{86}$ & $(0.15,0.05,0.8)$ & $(0.3,0.01,0.69)$ & $(0.1,0.05,0.85)$ & $(0.4,0,0.5)$ & $(0.2,0.1,0.6)$ & $(0.188,0.019,0.793)$ \\
\hline \multicolumn{2}{|c|}{ Similarity degree } & 0.582 & 0.647 & 0.633 & 0.56 & 0.653 & \\
\hline \multicolumn{2}{|c|}{ Expert weight } & 0.188 & 0.211 & 0.205 & 0.18 & 0.216 & \\
\hline \multicolumn{2}{|c|}{ Consensus degree } & 0.355 & 0.409 & 0.375 & 0.422 & 0.214 & \\
\hline
\end{tabular}


For the gene regulatory network, the truth degrees of the starting places are set as follows:

$$
\widetilde{\alpha}\left(p_{1}\right)=(0.742,0.108,0.06), \widetilde{\alpha}\left(p_{2}\right)=(0.878,0,0.06), \widetilde{\alpha}\left(p_{3}\right)=(0.45,0.305,0.194),
$$

$\widetilde{\alpha}\left(p_{4}\right)=(0.408,0.204,0.287), \widetilde{\alpha}\left(p_{5}\right)=(0.608,0.19,0.110), \widetilde{\alpha}\left(p_{6}\right)=(0.754,0.095,0.110)$.

With the PFPNs established in Figure 4, we can obtain:

$$
I=\left[\begin{array}{llllll}
1 & 0 & 0 & 0 & 0 & 0 \\
1 & 0 & 0 & 0 & 0 & 0 \\
1 & 0 & 0 & 0 & 0 & 0 \\
0 & 1 & 0 & 0 & 0 & 0 \\
0 & 0 & 1 & 0 & 0 & 0 \\
0 & 0 & 0 & 1 & 0 & 0 \\
0 & 0 & 0 & 0 & 1 & 0 \\
0 & 0 & 0 & 0 & 0 & 1 \\
0 & 0 & 0 & 0 & 0 & 0
\end{array}\right] O=\left[\begin{array}{llllll}
0 & 0 & 0 & 0 & 0 & 0 \\
0 & 0 & 0 & 0 & 0 & 0 \\
0 & 0 & 0 & 0 & 0 & 0 \\
0 & 0 & 0 & 0 & 0 & 0 \\
0 & 0 & 0 & 0 & 0 & 0 \\
0 & 0 & 0 & 0 & 0 & 0 \\
1 & 0 & 0 & 0 & 0 & 0 \\
0 & 1 & 1 & 1 & 0 & 0 \\
0 & 0 & 0 & 0 & 1 & 1
\end{array}\right]
$$

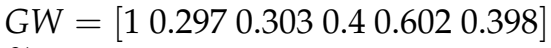

$\widetilde{U}=[(0.966,0,0)(0.946,0,0.02)(0.907,0,0)(0.988,0,0)(0.974,0,0.02)(0.988,0,0)]$

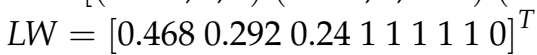

$\widetilde{T h}=[(0.196,0.2,0.604)(0.252,0.086,0.558)(0.12,0.202,0.599)(0.186,0.025,0.789)$

$(0.243,0.106,0.547)(0.106,0.184,0.608)(0.196,0.019,0.785)(0.188,0.019,0.793)(1,0,0)]^{T}$

$\widetilde{M}_{0}=[(0.742,0.108,0.06)(0.878,0,0.06)(0.45,0.305,0.194)(0.408,0.204,0.287)$

$$
(0.608,0.19,0.110)(0.754,0.095,0.110)(0,0,1)(0,0,1)(0,0,1)]^{T}
$$

Based on the concurrent inference algorithm of PFPNs, the reasoning process for the considered system is explained below.

(1) The enabled place vector $D^{(1)}$ is calculated using Equation (25) as follows:

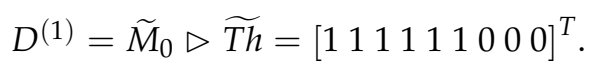

(2) The token value vector of input places $\widetilde{\Gamma}^{(1)}$ is calculated using Equation (26) as follows:

$$
\begin{aligned}
\widetilde{\Gamma}^{(1)} & =\operatorname{PFWG}\left((I \circ W)^{T}, \widetilde{M}_{0}\right) \\
& =[(0.834,0,0.094)(0.408,0.204,0.287)(0.608,0.19,0.11)(0.754,0.095,0.11)(0,0,1)(0,0,1)] .
\end{aligned}
$$

(3) The enabled transition vector $F^{(1)}$ is calculated using Equation (27) as follows:

$$
F^{(1)}=(E \times I) \triangleright\left(\left(D^{(1)}\right)^{T} \times I\right)=\left[\begin{array}{llllll}
1 & 1 & 1 & 1 & 0 & 0
\end{array}\right] .
$$

(4) The output truth degree vector $\Psi^{(1)}$ is calculated using Equation (28) as follows:

$$
\begin{aligned}
\Theta^{(1)} & =\left(F^{(1)} \circ \Gamma^{(1)}\right) \circ \widetilde{U} \\
& =[(0.8006,0,0.094)(0.587,0,0.287)(0.7182,0,0.11)(0.8405,0,0.11)(0,0,1)(0,0,1)] .
\end{aligned}
$$

(5) The new marking vector $\widetilde{M}_{1}$ is calculated using Equation (29) as follows:

$$
\begin{aligned}
\tilde{M}_{1}= & \tilde{M}_{0} \oplus \operatorname{PFWG}\left(\left(O \circ W_{g w}\right), \Theta^{(1)}\right) \\
= & {[(0.742,0.108,0.06)(0.878,0,0.06)(0.45,0.305,0.194)(0.408,0.204,0.287)} \\
& (0.608,0.19,0.110)(0.754,0.095,0.110)(0.801,0,0.094)(0.72,0,0.168)(0,0,1)]^{T} .
\end{aligned}
$$

(6) Since $\widetilde{M}_{1} \neq \widetilde{M}_{0}$, we will continue to next iteration and let $k=2$.

$$
\begin{aligned}
& D^{(2)}=\left[\begin{array}{lllllllll}
1 & 1 & 1 & 1 & 1 & 1 & 1 & 1 & 0
\end{array}\right]^{T} \\
& \widetilde{\Gamma}^{(2)}=[(0.834,0,0.094)(0.408,0.204,0.287)(0.608,0.19,0.11)(0.754,0.095,0.11) \\
& (0.801,0,0.094)(0.72,0,0.168)] \\
& F^{(2)}=\left[\begin{array}{llllll}
1 & 1 & 1 & 1 & 1 & 1
\end{array}\right] \\
& \Theta^{(2)}=[(0.8006,0,0.094)(0.587,0,0.287)(0.7182,0,0.11)(0.8405,0,0.11) \\
& (0.793,0,0.094)(0.7056,0,0.168)]
\end{aligned}
$$




$$
\begin{aligned}
\widetilde{M}_{2}= & {[(0.742,0.108,0.06)(0.878,0,0.06)(0.45,0.305,0.194)(0.408,0.204,0.287)} \\
& (0.608,0.19,0.110)(0.754,0.095,0.110)(0.801,0,0.094)(0.72,0,0.168)(0.757,0,0.125)]^{T} .
\end{aligned}
$$

(7) Since $\widetilde{M}_{2} \neq \widetilde{M}_{1}$, we will continue to next iteration, and $k=3$.

$$
\begin{aligned}
\widetilde{M}_{3}= & {[(0.742,0.108,0.06)(0.878,0,0.06)(0.45,0.305,0.194)(0.408,0.204,0.287)} \\
& (0.608,0.19,0.110)(0.754,0.095,0.110)(0.801,0,0.094)(0.72,0,0.168)(0.757,0,0.125)]^{T} .
\end{aligned}
$$

Since $\widetilde{M}_{3}=\widetilde{M}_{2}$, the reasoning process is over. The final PFNs of all the places are obtained as $\widetilde{M}_{3}$. The expression level of the target gene $d_{9}$ is $(0.757,0,0.125)$, which means that the degree of positive membership is 0.757 , the degree of neutral membership is 0 , and the degree of negative membership is 0.125 .

\subsection{Comparisons and Discussion}

To show the effectiveness of the proposed PFPNs, a comparison analysis with the IFPNs [40] and the conventional FPNs [34] are made in this part. The expression level of the target gene $d_{9}$ by using the IFPNs is $(0.725,0.149)$ [40]. For the given gene regulatory network, the knowledge parameters in the FPNs are shown as follows:

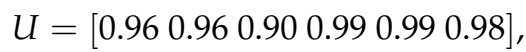

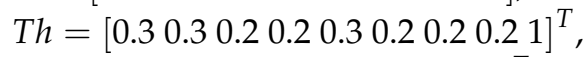

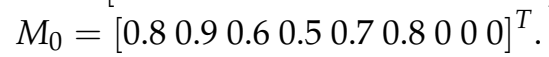

Based on the reasoning algorithm of FPNs, the result is obtained as:

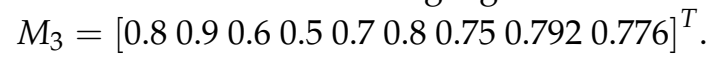

According to above three FPN models, the ranking results of the intermediate places $p_{7}, p_{8}$, and the terminating place $p_{9}$ are listed in Table 7 . First, we can find that the ranking results of PFPNs and IFPNs are the same. This can prove the feasibility of the proposed PFPNs, but IFSs are utilized in the IFPN model to handle uncertainty and vagueness in knowledge representation and reasoning. Although IFSs have been successfully applied in various areas, there are situations that cannot be represented by IFSs [19]. As a generalization of IFSs, the PFSs that consider the degree of positive membership, the degree of neutral membership, and the degree of negative membership are more suitable to describe uncertain information and data. For example, the expression level of the target gene $d_{9}$ is $(0.757,0,0.125)$ in PFPNs and $(0.725,0.149)$ in IFPNs. The neutral membership degree is ignored in the IFPNs. Thus, the proposed PFPNs have a wider range of applicability than the IFPNs.

Table 7. Rankings of $p_{7}, p_{8}$, and $p_{9}$ by PFPNs, intuitionistic FPNs (IFPNs), and FPNs.

\begin{tabular}{cc}
\hline FPN Models & Ranking Results \\
\hline PFPNs & $p_{7}>p_{9}>p_{8}$ \\
IFPNs & $p_{7}>p_{9}>p_{8}$ \\
FPNs & $p_{8}>p_{9}>p_{7}$ \\
\hline
\end{tabular}

The ranking results derived by the PFPNs and the FPNs are different. The main reason is that the information concerning neutral membership degree and negative membership degree is ignored when the FPN model is used. Thus, the original information will be lost in the knowledge representation and acquisition processes. Furthermore, the global weights are not taken into consideration in the traditional FPNs. This implies a lack of precision in the final reasoning result of FPNs.

In addition, the proposed model considers the conflict and inconsistency among expert evaluations in acquiring knowledge parameters. If conflicts and inconsistencies among experts are ignored, i.e., the five experts are treated equally, then the knowledge parameters are obtained as:

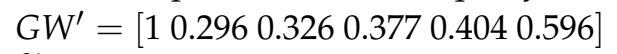

$\widetilde{U}^{\prime}=[(0.965,0,0)(0.945,0,0.021)(0.909,0,0)(0.988,0,0)(0.973,0,0.021)(0.988,0,0)]$

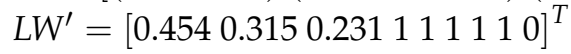


$\begin{aligned} \widetilde{T h^{\prime}}= & {[(0.379,0.197,0.371)(0.286,0.087,0.521)(0.136,0.204,0.583)(0.209,0.042,0.728)} \\ & (0.247,0.109,0.54)(0.146,0.184,0.562)(0.255,0.076,0.619)(0.257,0.0,0.715)(1,0,0)]^{T} .\end{aligned}$

Then the reasoning result of PFPNs is obtained as:

$$
\begin{aligned}
\tilde{M}_{3}^{\prime}= & {[(0.742,0.108,0.06)(0.878,0,0.06)(0.45,0.305,0.194)(0.408,0.204,0.287)} \\
& (0.608,0.19,0.110)(0.754,0.095,0.110)(0.807,0,0.093)(0.717,0,0.172) \\
& (0.739,0,0.148)]^{T} .
\end{aligned}
$$

Comparing $\widetilde{M}_{3}^{\prime}$ with $\widetilde{M}_{3}$, it is evident that the obtained expression levels of the target gene $d_{9}$ are different. This difference can be explained by the fact that the knowledge parameters acquired without considering the conflict between expert evaluations are inaccurate. For example, Table 8 shows the global weights derived with and without considering conflict evaluations of the experts (Case 1 and Case 2). In addition, the global weights acquired in the IFPNs [40] are presented as Case 3. It can be found that the global weights determined in the PFPNs that consider conflict and inconsistency are consistent with the original ones yielded in the IFPNs. Therefore, it is significant to take conflict and inconsistency among experts into account in the knowledge acquisition, and the knowledge parameters obtained in our proposed PFPNs are more reasonable and reliable.

Table 8. Global weights with respect to the considered cases.

\begin{tabular}{cccc}
\hline Global Weights & Case 1 & Case 2 & Case 3 \\
\hline$g w_{1}$ & 1 & 1 & 1 \\
$g w_{2}$ & 0.297 & 0.296 & 0.3 \\
$g w_{3}$ & 0.303 & 0.326 & 0.3 \\
$g w_{4}$ & 0.4 & 0.377 & 0.4 \\
$g w_{5}$ & 0.602 & 0.404 & 0.6 \\
$g w_{6}$ & 0.398 & 0.596 & 0.4 \\
\hline
\end{tabular}

In summary, the PFPNs proposed in this study have the following advantages. First, using PFSs, the FPPNs are more efficient in dealing with the vagueness and imprecision in knowledge representation. Second, via a similarity degree-based expert weighting method, the conflict and inconsistency among expert evaluations can be handled in knowledge acquisition. As a result, the knowledge parameters in PFPNs could be determined accurately based on the opinions of different experts. Third, compared with the reachability tree-based reasoning algorithm in current FPNs, the developed inference algorithm of PFPNs adopts a matrix equation format and can execute knowledge reasoning more efficiently.

\section{Conclusions}

In this article, a new type of FPNs, called PFPNs, is proposed to enhance the ability of FPNs in knowledge representation and acquisition. In modeling a rule-based system, the proposed model can deal with imprecise knowledge information by using PFSs. Considering the conflict and inconsistency among expert evaluations, a similarity degree-based method is adopted to objectively derive the weights of experts in the process of knowledge acquisition. Finally, a practical case of a gene regulatory network is presented to illustrate the applicability and usefulness of the proposed PFPNs. The results showed that the PFPN model proposed in this paper can overcome certain disadvantages of the traditional FPNs, and it is efficient for knowledge representation and acquisition.

In future research, other knowledge parameters, such as time factor, can be introduced into the PFPNs for time-critical systems. Second, it is interesting to apply the proposed model to more complex systems in future studies to further show its effectiveness and efficiency. Additionally, considerable computations are involved in the proposed PFPNs. Therefore, it is suggested to develop a software tool in the future for supporting the use and implementation of the PFPN model by engineers in practice. 
the abstract, introduction, research design, research methodology, and findings, and revised the manuscript. The discussion was a team task. All authors have read and approved the final manuscript.

Funding: This work was partially supported by the National Natural Science Foundation of China (Nos. 61773250, 71402090 and 71701153) and the Shanghai Youth Top-Notch Talent Development Program.

Acknowledgments: The authors are very grateful to the editor and reviewers for their insightful and constructive comments and suggestions, which are very helpful in improving the quality of the paper.

Conflicts of Interest: The authors declare no conflict of interest.

\section{References}

1. Chen, S.M. Weighted fuzzy reasoning using weighted fuzzy Petri nets. IEEE. Trans. Knowl. Data Eng. 2002, 14, 386-397. [CrossRef]

2. Yeung, D.S.; Tsang, E.C.C. Fuzzy knowledge representation and reasoning using Petri nets. Expert Syst. Appl. 1994, 7, 281-289. [CrossRef]

3. Zhou, K.Q.; Mo, L.P.; Jin, J.; Zain, A.M. An equivalent generating algorithm to model fuzzy Petri net for knowledge-based system. J. Intell. Manuf. 2017. [CrossRef]

4. Liu, H.C.; Xue, L.; Li, Z.W.; Wu, J. Linguistic Petri nets based on cloud model theory for knowledge representation and reasoning. IEEE. Trans. Knowl. Data Eng. 2018, 30, 717-728. [CrossRef]

5. Zhou, K.Q.; Gui, W.H.; Mo, L.P.; Zain, A.M. A bidirectional diagnosis algorithm of fuzzy Petri net using inner-reasoning-path. Symmetry 2018, 10, 192. [CrossRef]

6. Liu, H.C.; Lin, Q.L.; Ren, M.L. Fault diagnosis and cause analysis using fuzzy evidential reasoning approach and dynamic adaptive fuzzy Petri nets. Comput. Ind. Eng. 2013, 66, 899-908. [CrossRef]

7. Zhang, Y.; Zhang, Y.; Wen, F.; Chung, C.Y.; Tseng, C.L.; Zhang, X.; Zeng, F.; Yuan, Y. A fuzzy Petri net based approach for fault diagnosis in power systems considering temporal constraints. Int. J. Electr. Power Energy Syst. 2016, 78, 215-224. [CrossRef]

8. Ding, Z.; Zhou, Y.; Zhou, M. Modeling self-adaptive software systems by fuzzy rules and Petri nets. IEEE Trans. Fuzzy Syst. 2018, 26, 967-984. [CrossRef]

9. Hong, Z.; Feng, Y.; Li, Z.; Tian, G.; Tan, J. Reliability-based and cost-oriented product optimization integrating fuzzy reasoning Petri nets, interval expert evaluation and cultural-based DMOPSO using crowding distance sorting. Appl. Sci. 2017, 7, 791. [CrossRef]

10. Hamed, R.I.; Ahson, S.I.; Parveen, R. Designing genetic regulatory networks using fuzzy Petri nets approach. Int. J. Automat. Comput. 2010, 7, 403-412. [CrossRef]

11. Liu, F.; Chen, S.; Heiner, M.; Song, H. Modeling biological systems with uncertain kinetic data using fuzzy continuous Petri nets. BMC Syst. Biol. 2018, 12, 42. [CrossRef] [PubMed]

12. Hamed, R.I.; Ahson, S.I. Confidence value prediction of DNA sequencing with Petri net model. J. King Saud Univ.-Comput. Inf. Sci. 2011, 23, 79-89. [CrossRef]

13. Zhou, K.Q.; Zain, A.M. Fuzzy Petri nets and industrial applications: A review. Artif. Intell. Rev. 2016, 45, 405-446. [CrossRef]

14. Liu, H.C.; You, J.X.; Li, Z.W.; Tian, G. Fuzzy Petri nets for knowledge representation and reasoning: A literature review. Eng. Appl. Artif. Intell. 2017, 60, 45-56. [CrossRef]

15. Li, H.; You, J.X.; Liu, H.C.; Tian, G. Acquiring and sharing tacit knowledge based on interval 2-tuple linguistic assessments and extended fuzzy Petri nets. Int. J. Uncertain Fuzzy Knowl.-Based Syst. 2018, 26, 43-65. [CrossRef]

16. Liu, H.C.; You, J.X.; You, X.Y.; Su, Q. Fuzzy Petri nets using intuitionistic fuzzy sets and ordered weighted averaging operators. IEEE Trans. Cybern. 2016, 46, 1839-1850. [CrossRef] [PubMed]

17. Suraj, Z. A new class of fuzzy Petri nets for knowledge representation and reasoning. Fund. Inform. 2013, 128, 193-207.

18. Cuong, B.C. Picture fuzzy sets. J.Comput. Sci. Cybern. 2014, 30, 409-420.

19. Wei, G. Picture fuzzy Hamacher aggregation operators and their application to multiple attribute decision making. Fund. Inform. 2018, 157, 271-320. [CrossRef]

20. Zhang, X.Y.; Wang, X.K.; Yu, S.M.; Wang, J.Q.; Wang, T.L. Location selection of offshore wind power station by consensus decision framework using picture fuzzy modelling. J. Clean. Prod. 2018, 202, 980-992. [CrossRef] 
21. Son, L.H.; Van Viet, P.; Van Hai, P. Picture inference system: A new fuzzy inference system on picture fuzzy set. Appl. Intell. 2017, 46, 652-669. [CrossRef]

22. Wang, R.; Wang, J.; Gao, H.; Wei, G. Methods for MADM with picture fuzzy Muirhead mean operators and their application for evaluating the financial investment risk. Symmetry 2019, 11, 6. [CrossRef]

23. Yeung, D.S.; Tsang, E.C.C. Weighted fuzzy production rules. Fuzzy Sets Syst. 1997, 88, 299-313. [CrossRef]

24. Liu, H.C.; Liu, L.; Lin, Q.L.; Liu, N. Knowledge acquisition and representation using fuzzy evidential reasoning and dynamic adaptive fuzzy Petri nets. IEEE Trans. Cybern. 2013, 43, 1059-1072. [CrossRef] [PubMed]

25. Tang, Y. Learning-based disassembly process planner for uncertainty management. IEEE Trans. Syst. Man Cybern. A 2009, 39, 134-143. [CrossRef]

26. Tsang, E.C.; Yeung, D.S.; Lee, J.W.; Huang, D.M.; Wang, X.Z. Refinement of generated fuzzy production rules by using a fuzzy neural network. IEEE Trans. Syst. Man Cybern. B 2004, 34, 409-418. [CrossRef]

27. Liu, H.C.; You, J.X.; Tian, G. Determining truth degrees of input places in fuzzy Petri nets. IEEE Trans. Syst. Man Cybern. Syst. 2017, 47, 3425-3431. [CrossRef]

28. Zadeh, L.A. Fuzzy sets. Inf. Control 1965, 8, 338-353. [CrossRef]

29. Atanassov, K. Intuitionistic fuzzy sets. Fuzzy Sets Syst. 1986, 20, 87-96. [CrossRef]

30. Wei, G. Some similarity measures for picture fuzzy sets and their applications. Iranian J. Fuzzy Syst. 2018, 15, 77-89.

31. Wei, G. Picture fuzzy aggregation operators and their application to multiple attribute decision making. J. Intell. Fuzzy Syst. 2017, 33, 713-724. [CrossRef]

32. Garg, H. Some Picture Fuzzy Aggregation Operators and Their Applications to Multicriteria Decision-Making. Arab. J. Sci. Eng. 2017, 42, 5275-5290. [CrossRef]

33. Looney, C.G. Fuzzy Petri nets for rule-based decision-making. IEEE Trans. Syst. Man Cybern. 1988, 18, 178-183. [CrossRef]

34. Chen, S.M.; Ke, J.S.; Chang, J.F. Knowledge representation using fuzzy Petri nets. IEEE Trans. Knowl. Data Eng. 1990, 2, 311-319. [CrossRef]

35. Yeung, D.S.; Ysang, E.C.C. A multilevel weighted fuzzy reasoning algorithm for expert systems. IEEE Trans. Syst. Man Cybern. A 1998, 28, 149-158. [CrossRef]

36. Markiewicz, M.; Gniewek, L. A program model of fuzzy interpreted Petri net to control discrete event systems. Appl. Sci. 2017, 7, 422. [CrossRef]

37. Liu, H.C.; Lin, Q.L.; Mao, L.X.; Zhang, Z.Y. Dynamic adaptive fuzzy Petri nets for knowledge representation and reasoning. IEEE Trans. Syst. Man Cybern. Syst. 2013, 43, 1399-1410. [CrossRef]

38. Ha, M.H.; Li, Y.; Wang, X.F. Fuzzy knowledge representation and reasoning using a generalized fuzzy Petri net and a similarity measure. Soft Comput. 2007, 11, 323-327. [CrossRef]

39. Gao, M.M.; Zhou, M.C.; Huang, X.G.; Wu, Z.M. Fuzzy reasoning Petri nets. IEEE Trans. Syst. Man Cybern. A 2003, 33, 314-324.

40. Hamed, R.I. Quantitative modeling of gene networks of biological systems using fuzzy Petri nets and fuzzy sets. J. King Saud Univ.-Sci. 2018, 30, 112-119. [CrossRef]

(C) 2019 by the authors. Licensee MDPI, Basel, Switzerland. This article is an open access article distributed under the terms and conditions of the Creative Commons Attribution (CC BY) license (http://creativecommons.org/licenses/by/4.0/). 